\title{
Phonon confinement and self-limiting oxidation effect of silicon nanowires synthesized by laser ablation
}

\author{
N. Fukata ${ }^{a), b)}$ \\ Advanced Electronic Materials Center, National Institute for Materials Science, 1-1 Namiki, Tsukuba, \\ 305-0044, Japan \\ T. Oshima, N. Okada, and K. Murakami ${ }^{\text {b) }}$ \\ Institute of Applied Physics, University of Tsukuba, 1-1-1 Tennôdai, Tsukuba 305-8573, Japan \\ T. Kizuka \\ Institute of Materials Science, University of Tsukuba, 1-1-1 Tennôdai, Tsukuba 305-8573, Japan \\ T. Tsurui and S. Ito \\ Institute for Materials Research, Tohoku University, Sendai 980-8577, Japan
}

(Received 8 November 2005; accepted 6 May 2006; published online 25 July 2006)

The phonon confinement and self-limiting oxidation effects of silicon nanowires (SiNWs) synthesized by laser ablation were investigated. The size of SiNWs was controlled by the synthesis parameters during laser ablation and the subsequent thermal oxidation. Thermal oxidation increases the thickness of the SiNWs' surface oxide layer, resulting in a decrease in their crystalline Si core diameter. This effect causes a downshift and asymmetric broadening of the Si optical phonon peak due to phonon confinement, while excess oxidation causes an upshift due to compressive stress. The compressive stress retarded the oxidation of the SiNWs by self-limiting oxidation effect. This result shows that the Si core diameter can be controlled by compressive stress. () 2006 American Institute of Physics. [DOI: 10.1063/1.2218386]

\section{INTRODUCTION}

One-dimensional semiconductor nanowires such as silicon nanowires (SiNWs) are of great importance due to their interesting physical properties and potential for application as electronic and optical devices, ${ }^{1-3}$ bio/chemical sensors, ${ }^{4}$ and probe microscopy tips. ${ }^{5}$ Furthermore, it has proved possible to realize nanomanipulators if SiNWs can be created according to design. ${ }^{6}$

In order to realize the above-mentioned applications, it is important to investigate size control and the quantum size effects that vary with their diameters. Generally, SiNWs are synthesized by laser ablation ${ }^{7-10}$ or chemical vapor deposition (CVD). ${ }^{11,12}$ In the latter case, the diameter can be controlled by the size of the metal catalyst particles which are placed in advance. This growth mechanism is called the vapor-liquid-solid (VLS) mechanism. ${ }^{13,14}$ On the other hand, the advantage of the former method is that SiNWs can be synthesized in the gas phase without forming or placing metal catalysts of nanometer size as seeds for SiNWs. Not using nanometer-scale metal catalysts, however, does not allow the size and location of SiNWs to be controlled effectively.

Size control of SiNWs is important for studying the effects of quantum confinement, ${ }^{15}$ which is one of the unique physical properties of nanostructures. Until recently, a blueshift in the photoluminescence peak, ${ }^{16}$ increased electronic band gap, ${ }^{17}$ and shifts of the optical phonon peak of Si (Refs.

\footnotetext{
a) Electronic mail: fukata.naoki@nims.go.jp

${ }^{b}$ Also at Special Research Project on Nanoscience, University of Tsukuba, 1-1-1 Tennôdai, Tsukuba 305-8573, Japan.
}

10 and 18-20) have been observed for SiNWs with decreasing diameter. Adu et al. investigated the phonon confinement effect in SiNWs with different diameters and observed a downshift and broadening of the Si optical phonon peak. ${ }^{20}$ More recently, we have also investigated the phonon confinement effect of continuously thermally oxidized SiNWs. ${ }^{10,21}$ Thermal oxidation of the SiNWs clearly resulted in a gradual downshift and broadening of the Si optical phonon peak as a result of a stepwise decrease in the diameter of the SiNWs. Furthermore, excess oxidation produced an opposite change to the phonon confinement effect; namely, an upshift of the Si optical phonon peak due to compressive stress. In this study, the effect of the surface oxide layer on stress was also investigated, since the Si core diameter of the SiNWs can be self-limited by it. Here, the formation of a highly stressed oxide increases in the activation energy of oxidant diffusivity and a resultant decrease in the oxidation rate. $^{22,23}$ This effect is called self-limiting oxidation. Until now, this effect has been investigated only in lithographically formed Si nanocolumns. ${ }^{22,23}$ No experiments have been carried out up to now on SiNWs synthesized using a bottom-up method such as laser ablation.

In the present study, first, to achieve the size control of SiNWs even when using laser ablation, we synthesized SiNWs under various conditions and investigated the dependence on the synthesis parameters such as content of catalyst, synthesis temperature, laser power, and pressure of flowing gas during laser ablation. The phonon confinement effect that depends on the diameter of the SiNWs was investigated by using thermally oxidized SiNWs or SiNWs synthesized under different conditions. Self-limiting oxidation due to accu- 
mulated stress was also investigated in the thermally oxidized SiNWs to investigate the feasibility of controlling the Si core diameter of SiNWs.

\section{EXPERIMENT}

SiNWs were synthesized by laser ablation of a Si target with a nickel $(\mathrm{Ni})$ or an iron $(\mathrm{Fe})$ catalyst. Four kinds of $\mathrm{Si}-\mathrm{Ni}$ targets, namely, $\mathrm{Si}_{99.5} \mathrm{Ni}_{0.5}, \mathrm{Si}_{99} \mathrm{Ni}_{1}, \quad \mathrm{Si}_{95} \mathrm{Ni}_{5}$, and $\mathrm{Si}_{90} \mathrm{Ni}_{10}$, and four kinds of $\mathrm{Si}-\mathrm{Fe}$ targets, namely, $\mathrm{Si}_{99} \mathrm{Fe}_{1}$, $\mathrm{Si}_{98} \mathrm{Fe}_{2}, \mathrm{Si}_{95} \mathrm{Fe}_{5}$, and $\mathrm{Si}_{90} \mathrm{Fe}_{10}$ targets were used. Each target was placed in a quartz tube and heated to $1000-1200{ }^{\circ} \mathrm{C}$ in flowing argon (Ar) gas at 50 SCCM (SCCM denotes cubic centimeter per minute at STP). The temperatures were, respectively, determined from the eutectic point of $\mathrm{Si}-\mathrm{Ni}$ and $\mathrm{Si}-\mathrm{Fe}$ alloys. Ar gas pressures were set at 100, 300, 400, 500, 600 , and 700 Torr to investigate appropriate growth conditions, and almost all syntheses were carried out at 500 Torr. After reaching the designated temperature, a frequencydoubled Nd:YAG(yttrium aluminum garmet) laser (wavelength of $532 \mathrm{~nm}$ ) was focused on the target. To avoid continuous irradiation of the same position, the focused point was scanned during laser ablation. The laser power was mainly about $200 \mathrm{~mJ} /$ pulse and partly $100 \mathrm{~mJ} /$ pulse, with a pulse duration of $7 \mathrm{~ns}$ at $10 \mathrm{~Hz}$. In previous studies, ${ }^{7-9}$ SiNWs synthesized by laser ablation were collected on a water-cooled copper finger or the inner wall of a quartz tube enclosing the copper finger. It was therefore necessary to collect SiNWs from where they were deposited places after the synthesis. In this experiment, to improve this task, SiNWs were directly collected on a $\mathrm{Si}$ substrate which was placed on a water-cooled molybdenum holder. Owing to this, SiNWs could be observed while being deposited. A proportion of the specimens were thermally oxidized at temperatures from 700 to $1000{ }^{\circ} \mathrm{C}$ for $30 \mathrm{~min}$ in $\mathrm{O}_{2}$ gas at a pressure of 200 Torr to investigate the phonon confinement effect. Furthermore, to investigate the self-limiting oxidation of the SiNWs, isothermal oxidations were performed at 800 and $1000{ }^{\circ} \mathrm{C}$.

Scanning electron microscopy (SEM: JEOL, JSM-5610, $20 \mathrm{kV}$ ) and transmission electron microscopy (TEM: JEOL, JEM4000EX: $400 \mathrm{kV}$, JEM-2010: $200 \mathrm{kV}$ ) were used to observe $\mathrm{SiNWs}$ and to investigate their detailed structures. Micro-Raman scattering measurements were performed at room temperature $(\mathrm{RT})$ with a $100 \times$ objective and a $532 \mathrm{~nm}$ excitation light at 0.02 and $20 \mathrm{~mW}$.

\section{RESULTS}

\section{A. Size control of SiNWs}

A representative SEM image of SiNWs synthesized at $1200{ }^{\circ} \mathrm{C}$ using a $\mathrm{Si}_{99} \mathrm{Ni}_{1}$ target is shown in Fig. 1(a). Hereinafter, we call these SiNWs $1200{ }^{\circ} \mathrm{C}$ SiNWs. The image shows a large number of SiNWs deposited on a Si substrate. TEM images of 1000 and $1200{ }^{\circ} \mathrm{C}$ SiNWs are shown in Figs. 1(b) and 1(c), respectively. The TEM image in Fig. 1(c) is shown at high resolution in Fig. 1(d), which clearly shows the Si lattice fringes inside the SiNWs, indicating that the $\mathrm{SiNW}$ is sheathed with an amorphous $\mathrm{SiO}_{x}(x \leqslant 2)$ layer; the core is crystal $\mathrm{Si}$. The average $\mathrm{Si}$ core diameters of 1000 and

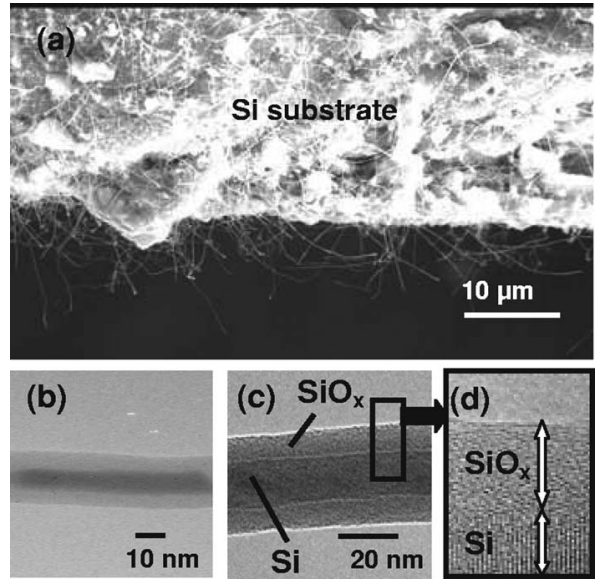

FIG. 1. (a) Representative SEM image of the SiNWs synthesized at $1200{ }^{\circ} \mathrm{C}$ and TEM images of the SiNWs synthesized at (b) 1000 and (c) $1200{ }^{\circ} \mathrm{C}$. A high-resolution TEM image of $1200^{\circ} \mathrm{C}$ synthesis $\left(1200^{\circ} \mathrm{C}\right.$ SiNWs) is shown in (d). The SiNWs were synthesized using $\mathrm{Si}_{99} \mathrm{Ni}_{1}$ targets.

$1200{ }^{\circ} \mathrm{C}$ SiNWs were about 10 and $17 \mathrm{~nm}$, respectively. The diameter of SiNWs is controllable by changing the synthesis parameters.

The dependences of the diameter of SiNWs on the synthesis temperature and the content of $\mathrm{Ni}$ catalyst are shown in Fig. 2. Here, the diameter of the SiNWs is the total diameter of the Si core and the surface oxide layer as estimated by SEM measurements. The synthesis temperatures were set at 1000,1100 , and $1200{ }^{\circ} \mathrm{C}$, since the eutectic point of $\mathrm{Si}-\mathrm{Ni}$ alloy is around $970{ }^{\circ} \mathrm{C}$. The diameter of the SiNWs closely depended on both the synthesis temperature and the content of Ni catalyst, i.e., it decreased with them. The dependence on the content of catalyst was also investigated in the case of
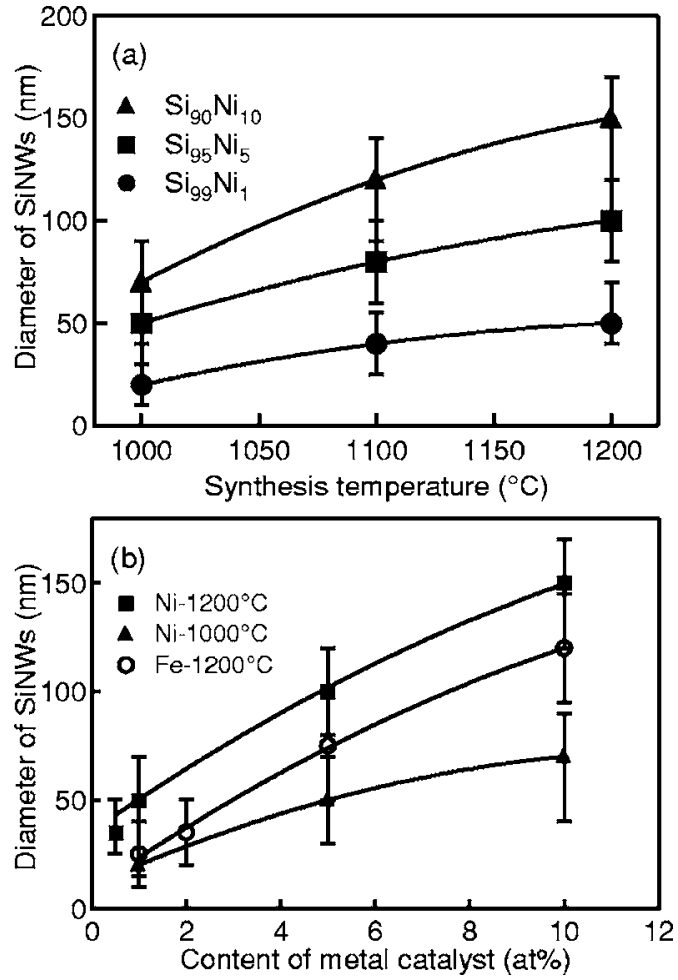

FIG. 2. Dependence of the diameter of the SiNWs on (a) synthesis temperature and (b) content of metal catalyst. 
Fe catalyst. The temperature was set at $1200{ }^{\circ} \mathrm{C}$, since the eutectic point of $\mathrm{Si}-\mathrm{Fe}$ alloy is around $120{ }^{\circ} \mathrm{C}$. The diameter decreased with decreasing content of Fe catalyst, similar to the result for the Ni catalyst, as shown in Fig. 1. The value obtained at $1200{ }^{\circ} \mathrm{C}$ in the case of the Ni catalyst was greater than that of $\mathrm{Fe}$ catalyst, showing that the diameter also depends on the type of metal catalyst. Hence, SiNWs with smaller diameters can be synthesized by decreasing the synthesis temperature and content of the metal catalyst.

Argon gas pressure also affects the growth of SiNWs, since it promotes the clustering of $\mathrm{Si}$ atoms and metal catalyst as seeds of SiNWs and also promotes the further addition of $\mathrm{Si}$ atoms that trigger the growth of SiNWs. Under our experimental conditions, SiNWs could be synthesized at Ar gas pressures ranging from 400 to 600 Torr. No SiNWs were observed at 100, 300, or 700 Torr. These results show that pressures lower than 400 Torr are not sufficient to form nanoclusters of $\mathrm{Si}$ and metal atoms, while pressures higher than 600 Torr promote further clustering, leading to the disturbance of one-dimensional growth by supersaturation of the clusters. An Ar gas pressure of 500 Torr was the most appropriate under our experimental conditions. We had previously investigated the dependence on laser power during laser ablation and found that average diameter decreases with decreasing the laser power. ${ }^{6}$

\section{B. Optical phonon in SiNWs}

\section{Dependence on synthesis temperature}

The phonon confinement effect depending on the diameter of the SiNWs was investigated by micro-Raman scattering measurements with two different excitation powers of 20 and $0.02 \mathrm{~mW}$, respectively. In the case of Raman measurements, SiNWs were collected not on $\mathrm{Si}$ wafers but on $\mathrm{SiO}_{2}$ substrates to avoid Si substrate effects and to observe only the Si optical phonons from the SiNWs. This method enabled us to deposit a large number of SiNWs at a time and to increase the Raman intensity, resulting in more precise analysis of peak shift and broadening of the Si optical phonon peak. In addition, we were able to apply thermal oxidation and HF treatment to the same SiNWs in the same specimen. A downshift and asymmetric broadening of the $\mathrm{Si}$ optical phonon peak of the SiNWs were observed in the Raman spectra shown in Fig. 3. The Si optical phonon peak shifted toward the lower frequencies with a decrease in the synthesis temperature, showing a decrease in the diameter of the SiNWs. The downshift observed for $0.02 \mathrm{~mW}$ excitation was about $1 \mathrm{~cm}^{-1}$ for the $1000{ }^{\circ} \mathrm{C}$ SiNWs. The Si optical phonon peak moved to lower frequencies and broadened as excitation laser power increased. The degrees of both the downshift and the peak broadening became increasingly dependent on the size of the SiNWs with increasing excitation power.

\section{Dependence on oxidation temperature}

The phonon confinement was also investigated for thermally oxidized SiNWs, as shown in Figs. 4 and 5. Thermal oxidation increases the thickness of the $\mathrm{SiO}_{x}(x \leqslant 2)$ layer and consequently decreases the diameter of the Si core. To
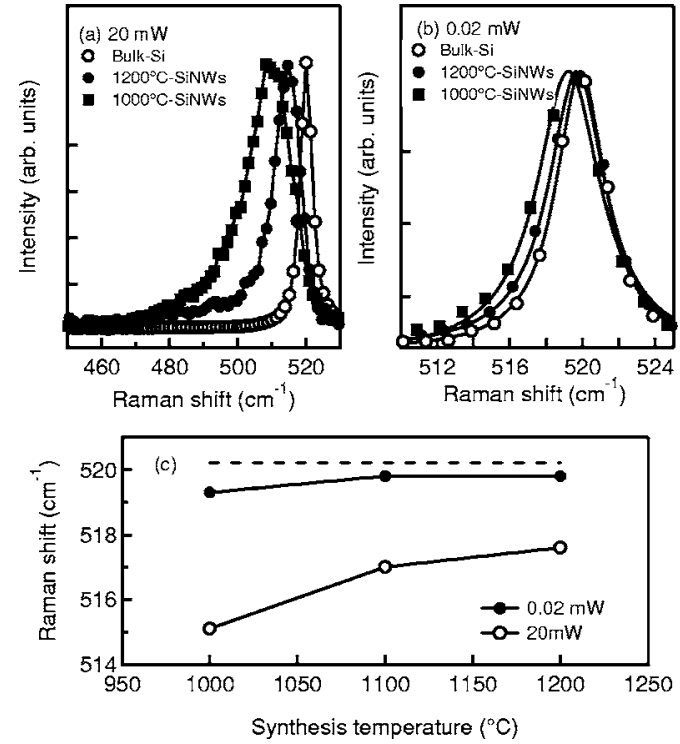

FIG. 3. Optical phonon peaks of the SiNWs measured by micro-Raman scattering at excitation powers of (a) 20 and (b) $0.02 \mathrm{~mW}$. (c) Dependence of the Raman shift of the Si optical phonon peak on synthesis temperature and excitation power. The SiNWs were synthesized using a $\mathrm{Si}_{99} \mathrm{Ni}_{1}$ target.

investigate the phonon confinement effect in SiNWs with different diameters, both the 1000 and $1200{ }^{\circ} \mathrm{C}$ SiNWs were thermally oxidized at temperatures from 700 to $1000^{\circ} \mathrm{C}$ for $30 \mathrm{~min}$ in $\mathrm{O}_{2}$ gas at a pressure of 200 Torr. The Raman measurements were performed at an excitation power of $0.02 \mathrm{~mW}$ to prevent local heating effects of irradiation by the excitation laser. This will be discussed in Sec. IV. The Si optical phonon peak slightly shifted towards lower frequen-
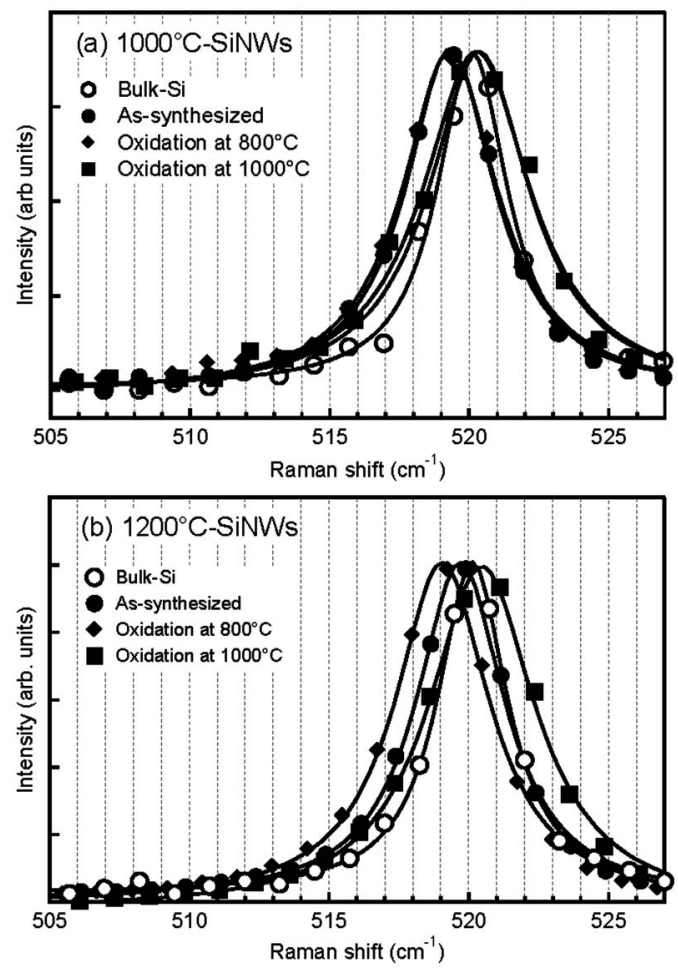

FIG. 4. Dependence on oxidation temperature of the Si optical phonon peak of the SiNWs synthesized at (a) 1000 and (b) $1200{ }^{\circ} \mathrm{C}$. The SiNWs were synthesized using a $\mathrm{Si}_{99} \mathrm{Ni}_{1}$ target. 

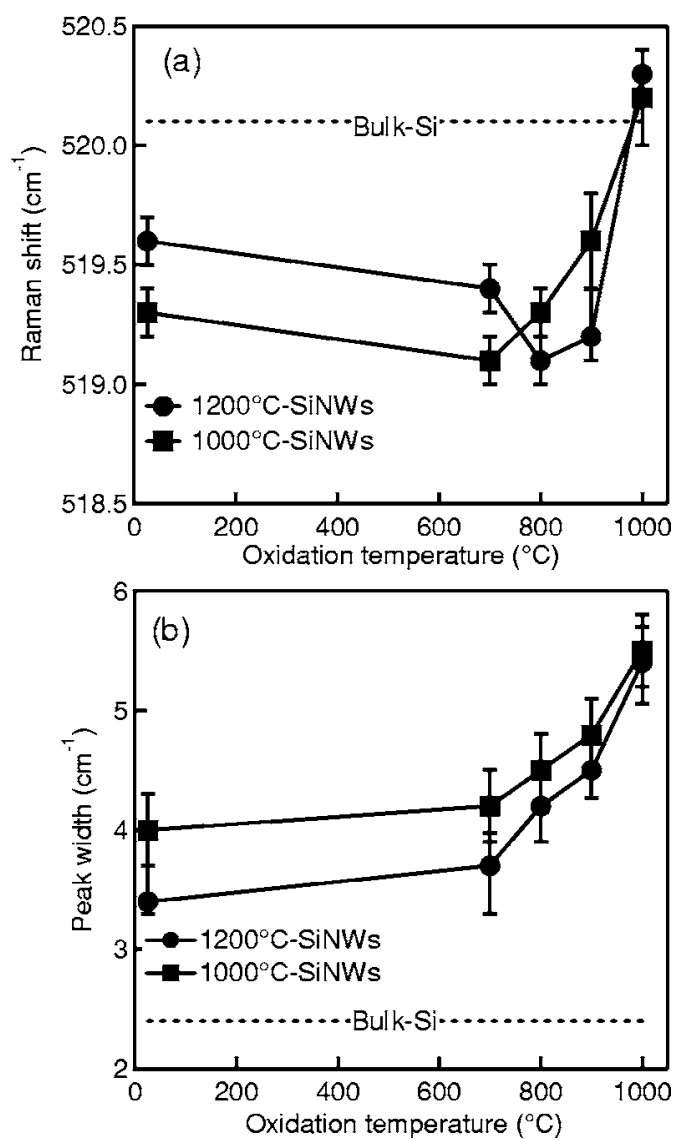

FIG. 5. Dependence on oxidation temperature of (a) Raman shift and (b) peak width of the Si optical phonon peak of the SiNWs synthesized at 1000 and $1200{ }^{\circ} \mathrm{C}$, respectively. The dotted lines show the values for bulk Si. The SiNWs were synthesized using a $\mathrm{Si}_{99} \mathrm{Ni}_{1}$ target.

cies and then shifted higher after thermal oxidation at above $800{ }^{\circ} \mathrm{C}$ for the $1200{ }^{\circ} \mathrm{C}$ SiNWs and $700{ }^{\circ} \mathrm{C}$ for the $1000{ }^{\circ} \mathrm{C}$ SiNWs. The downshift is due to phonon confinement, while the upshift is caused by the increased compressive stress due to the progress of surface oxidation. ${ }^{10,21}$ The optical phonon peak of the $1000{ }^{\circ} \mathrm{C} \mathrm{SiNWs}$ before and after thermal oxidation at $700{ }^{\circ} \mathrm{C}$ showed a lower shift than that seen in $1200{ }^{\circ} \mathrm{C}$ SiNWs. Furthermore, the upshift of the Si optical phonon peak of the $1000{ }^{\circ} \mathrm{C}$ SiNWs was observed from lower oxidation temperatures than $1200{ }^{\circ} \mathrm{C}$ SiNWs.

Figure 6(a) shows the diameter of the Si core of SiNWs estimated by TEM measurements as a function of the oxidation temperature. The representative TEM images observed for the as-synthesized and thermally oxidized SiNWs are shown in Figs. 6(b)-6(d), and an illustration of the oxidation of the SiNWs is shown in Fig. 6(e). The Si core diameter decreased with increasing oxidation temperature. Figure 6 also shows the ratio of the diameter of the SiNWs to that of the $\mathrm{Si}$ core of the SiNWs as a function of the oxidation temperature. The figure shows an abrupt increase at $1000{ }^{\circ} \mathrm{C}$.

\section{Dependence on oxidation time}

To investigate the self-limiting oxidation effect in SiNWs, the dependence of the optical phonon peak on thermal oxidation time was investigated at 800 and $1000{ }^{\circ} \mathrm{C}$ for
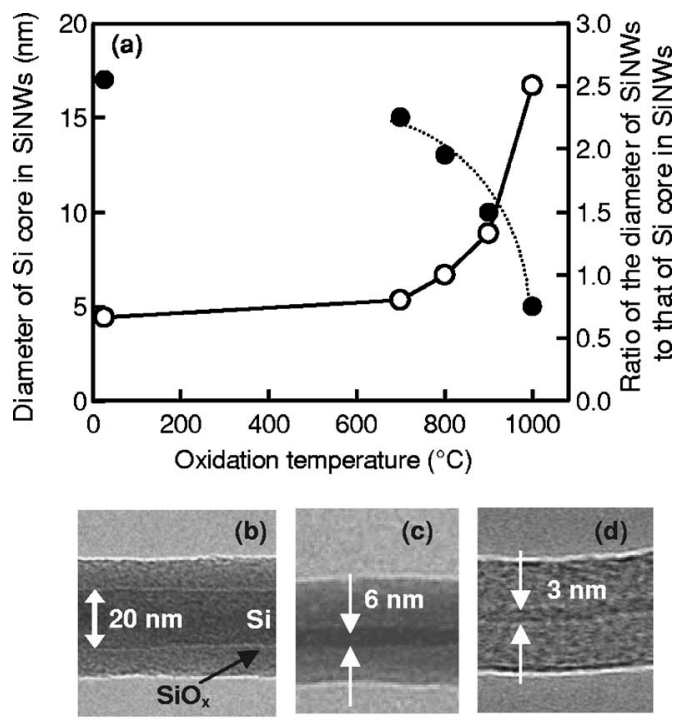

(e)

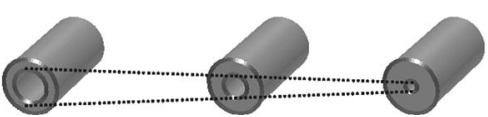

FIG. 6. (a) Diameter of the Si core of the SiNWs estimated by TEM measurements (closed circles) and the ratio of the diameter of the SiNWs to that of the Si core of the SiNWs (open circles) as a function of the oxidation temperature. Representative TEM images observed for (b) as-synthesized SiNWs and (c) the SiNWs thermally oxidized at $1000{ }^{\circ} \mathrm{C}$. (d) TEM image of the SiNWs with the minimum diameter observed after thermal oxidation at $1000{ }^{\circ} \mathrm{C}$ and (e) illustration of the oxidation of the SiNWs. The SiNWs were synthesized at $1200{ }^{\circ} \mathrm{C}$ using a $\mathrm{Si}_{99} \mathrm{Ni}_{1}$ target.

the $1200{ }^{\circ} \mathrm{C} \mathrm{SiNWs}$, as shown in Figs. $7(\mathrm{a})$ and $7(\mathrm{~b})$. In the case of oxidation at $800{ }^{\circ} \mathrm{C}$, the optical phonon peak first slightly shifted towards a lower wave number, then shifted higher, and finally reached a constant value. On the other hand, the optical phonon peak showed an increase even after oxidation at $1000{ }^{\circ} \mathrm{C}$ for $180 \mathrm{~min}$. The peak width also showed a different dependence on oxidation time between the case of $800{ }^{\circ} \mathrm{C}$ and that of $1000{ }^{\circ} \mathrm{C}$. In the former, it increased up to $60 \mathrm{~min}$ and reached an almost constant value, and in the latter, it showed an increase even after oxidation for $180 \mathrm{~min}$, as well as for the Raman shift. A representative TEM image of SiNWs thermally oxidized at $800{ }^{\circ} \mathrm{C}$ for 180 min is shown in Fig. 7(c), which shows that the Si core diameter of the SiNWs is self-limited at about $8 \mathrm{~nm}$ in this thermal oxidation condition.

\section{DISCUSSION}

\section{A. Growth of SiNWs}

The dependence of the diameter of the SiNWs on synthesis temperature can be explained as follows. The mechanism of VLS growth has been described in detail. ${ }^{7,8}$ In the case of VLS growth of SiNWs caused by laser ablation of $\mathrm{Si}$-metal targets, first, $\mathrm{Si}$ atoms and metal catalysts are evaporated by ablation. They form liquid nanoalloys by clustering due to the high pressure of the inert Ar gas. The role of metal catalysts is the key to forming liquid nanoalloys, which act as seeds of the SiNWs, and to controlling the diameter of the SiNWs. The growth of the SiNWs begins from the liquid nanoalloys after they become supersaturated 

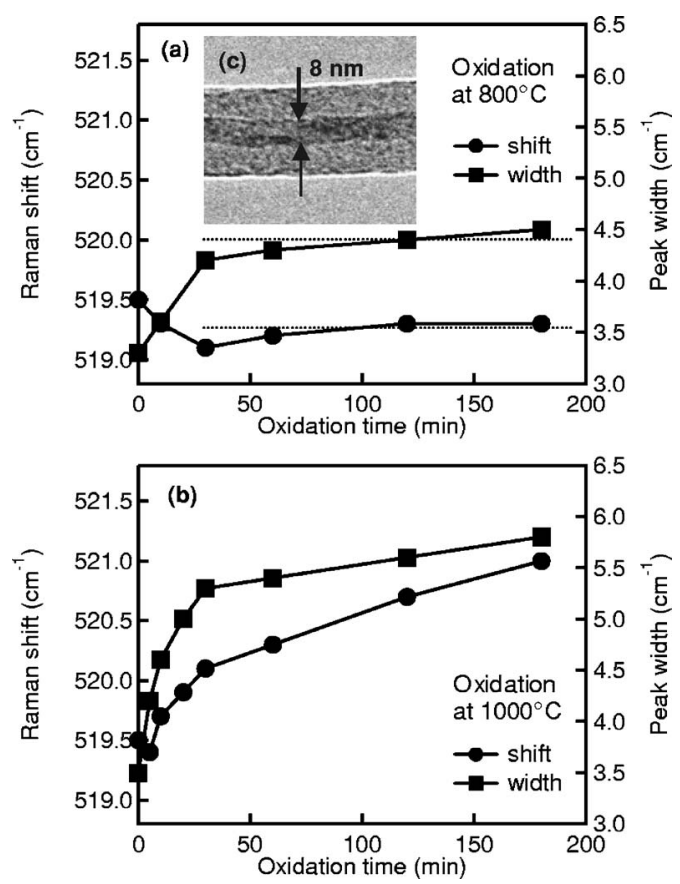

FIG. 7. Dependence of the Raman shift and the peak width of the Si optical phonon peak of the SiNWs on oxidation time. The synthesized temperature was set at $1200{ }^{\circ} \mathrm{C}$ and the following thermal oxidations were carried out at (a) 800 and (b) $1000^{\circ} \mathrm{C}$. (c) The inset is a TEM image of the SiNWs after thermal oxidation at $800^{\circ} \mathrm{C}$ for $180 \mathrm{~min}$.

in $\mathrm{Si}$. The supersaturation progress decelerates at higher temperatures, since nanoalloys can remain in a melted state for longer time. This leads to the formation of larger sizes of liquid nanoalloys and ultimately to an increase in the diameter of the SiNWs.

The dependence on the content of metal catalyst can be explained as follows. The volumes of the supersaturated liquid nanoalloys depend on the number of $\mathrm{Si}$ atoms which can dissolve into the liquid nanoalloy before supersaturation, i.e., it decreases with decreased metal catalyst in the liquid nanoalloys. Hence, the SiNWs' diameters decrease with decreased content of metal catalyst.

The diameter of SiNWs is also affected by the number of ablated atoms. The diameters of SiNWs depend on laser power. ${ }^{21}$ The number of ablated atoms increases with increased laser power. This affects the size of the supersaturated liquid nanoalloys and consequently the diameter of SiNWs increases with increased laser power. Based on these results, SiNWs with smaller diameters can be synthesized by decreasing the content of metal catalyst, synthesis temperature, and laser power during laser ablation.

\section{B. Phonon confinement effect in SiNWs}

The phonon confinement effect is one of the unique futures of low-dimensional structures. The phonons involved in first-order Raman scattering have $\mathbf{q} \sim 0$, where $\mathbf{q}$ is the wave vector of the scattered phonon. Finite-size effects or disorder partially relax momentum conservation and the selection rule is relaxed. This gives a downshift and an asymmetric broadening to the $\mathrm{Si}$ optical phonon peak. ${ }^{24,25}$

The downshift and asymmetric broadening of the Si optical phonon peak are shown in Figs. 3-5. As shown in Fig.
3, the Si optical phonon peak moves to lower frequencies and broadens as excitation laser power increases. As previously reported by Piscanec et al. ${ }^{18}$ this is caused by the local heating of SiNWs in Raman measurements. Furthermore, the degrees of both the downshift and broadening were much more dependent on the size of the SiNWs with increasing excitation power. This is caused by the difference in the thermal conductivity among SiNWs with different diameters. This result suggests that the effect of local heating also gives information on the diameter of the SiNWs and their thermal conductivities depending on the diameters. Based on these results, to investigate the phonon confinement effect, Raman measurements must be performed at very low excitation powers to avoid local heating of the SiNWs.

The results in Fig. 3 show that the Si optical phonon peak moves to lower frequencies and broadens as synthesis temperature decreases, namely, the diameter of the SiNWs decreases. The Si optical phonon peak also shifted towards lower wave number and broadened after thermal oxidation at $700-800{ }^{\circ} \mathrm{C}$, as shown in Figs. 4 and 5. In Fig. 5, the optical phonon peak of the $1000{ }^{\circ} \mathrm{C}$ SiNWs before and after thermal oxidation at $700{ }^{\circ} \mathrm{C}$ showed a lower shift than that seen in $1200{ }^{\circ} \mathrm{C}$ SiNWs. These are due to the increased phonon confinement in the SiNWs with decreasing Si core diameter.

According to the theoretical calculations based on the phonon confinement model of Richter et al. ${ }^{24}$ and Campbell and Fauchet, ${ }^{25}$ the Raman intensity is given by

$$
I(\omega)=\int \frac{|C(0, q)|^{2}}{[\omega-\omega(q)]^{2}+\left(\Gamma_{0} / 2\right)^{2}} d^{3} q,
$$

where $C(0, q)$ is a Fourier coefficient of the confinement function, $\omega(q)$ is Si phonon dispersion, and $\Gamma_{0}$ is the full width at half maximum of the reference Si. Here, we used the following relations, namely, $|C(0, q)|^{2}$ $=\exp \left(-q^{2} d^{2} / 16 \pi^{2}\right)$ and $\omega(q)=[A+B \cos (q \pi / 2)]^{0.5}+D$, with $A=1.714 \times 10^{15} \mathrm{~cm}^{-2}$ and $B=10^{5} \mathrm{~cm}^{-2} \cdot{ }^{18} D$ is an adjusting parameter to the bulk reference $\mathrm{Si}$ measured in this study. The value of $d$ corresponds to the diameter of the Si core of the SiNWs. Furthermore, SiNWs are not spherical nanoparticles but cylindrical nanowires with length $\geqslant$ diameter. Considering this, $d^{3} q$ in Eq. (1) can be simplified to $q d q$. By fitting the observed Raman spectra with Eq. (1), the diameters of the SiNWs were calculated to be about 15, 12, and $9 \mathrm{~nm}$ for the 1200,1100 , and $1000{ }^{\circ} \mathrm{C}$ SiNWs, respectively. Furthermore, the diameters of the $1200{ }^{\circ} \mathrm{C}$ SiNWs after thermal oxidation at 700 and $800{ }^{\circ} \mathrm{C}$ were also calculated to be about 13 and $10 \mathrm{~nm}$, respectively. In the case of the $1000{ }^{\circ} \mathrm{C}$ SiNWs after thermal oxidation at $700{ }^{\circ} \mathrm{C}$, the diameter was calculated to be about $8 \mathrm{~nm}$. These values correspond to the mean diameters of SiNWs without a surface oxide layer. The calculated values for the SiNWs were in good agreement with the values obtained by TEM observation in Fig. 6. Hence, the relationship between the downshift and broadening of the optical phonon peak and the diameter of SiNWs is more than adequately explained by the phonon confinement model. 


\section{Compressive stress and self-limiting oxidation in SiNWs}

Excess oxidation causes the upshift of the Si optical phonon peak. This can be explained by the increase in the compressive stress toward the center of the SiNWs, arising from the expansion of the surrounding surface oxide layer. ${ }^{10}$ The upshift of the Si optical phonon peak of the $1000{ }^{\circ} \mathrm{C}$ SiNWs was observed from lower oxidation temperatures than that seen in $1200{ }^{\circ} \mathrm{C}$ SiNWs, as shown in Fig. 5. This result shows that SiNWs with smaller core diameters are more sensitive to stress. The results shown in Fig. 6 show the diameter of the Si core of the SiNWs to decrease with increasing oxidation temperature, resulting in an abrupt increase in the ratio of the diameter of SiNWs to that of the Si core of the SiNWs after thermal oxidation at $1000{ }^{\circ} \mathrm{C}$, meaning that the compressive stress abruptly increases after thermal oxidation at $1000{ }^{\circ} \mathrm{C}$.

The effect of compressive stress on the frequency shift of the Si optical phonon peak has been already investigated by Mernagh and Liu in bulk Si. ${ }^{26}$ They precisely investigated the relation between the stress and the frequency shift of the Si optical phonon peak at RT for pressures up to $180 \mathrm{kbars}$ in a diamond-anvil cell. According to their results, the pressure dependence of the frequency shift is expressed by

$$
\Delta \omega=0.55 P-8.66 \times 10^{-4}\left(P^{2}\right),
$$

where $\Delta \omega$ is the frequency shift in $\mathrm{cm}^{-1}$ and $p$ is the pressure in kbars. Here, the frequency shift means the shift from the value of the bulk Si. On the other hand, the downshift due to phonon confinement effect should be considered for the estimation of stress in thermally oxidized SiNWs, i.e., the frequency shift is not from the value in bulk Si but from the value downshifted by the phonon confinement effect. This makes precise stress estimation difficult. If the frequency shift were from the lowest frequency obtained after thermal oxidation at $800{ }^{\circ} \mathrm{C}$ in the case of $1200{ }^{\circ} \mathrm{C}$ SiNWs and $700{ }^{\circ} \mathrm{C}$ in the case of $1000^{\circ} \mathrm{C}$ SiNWs, the compressive stress in the SiNWs after thermal oxidation at $1000^{\circ} \mathrm{C}$ can be roughly estimated to be about $200 \mathrm{MPa}$ in both cases.

The formation of such a highly stressed thick oxide layer around the SiNWs decreases the oxidation rate. ${ }^{22,23}$ This effect is called self-limiting oxidation. Figures 7(a) and 7(b) show the dependence of the Si optical phonon peak on oxidation time. The Raman shift and width of the Si optical phonon peak showed saturation after thermal oxidation at $800{ }^{\circ} \mathrm{C}$ for more than $60 \mathrm{~min}$. This is explained by the selflimiting oxidation effect caused by the compressive stress accumulated in the SiNWs. The compressive stress induced by thermal oxidation at $800{ }^{\circ} \mathrm{C}$ for $60-180 \mathrm{~min}$ is estimated to be about $70 \mathrm{MPa}$ by applying the same above-mentioned method. In Fig. 7(c), the TEM image obtained after thermal oxidation at $800{ }^{\circ} \mathrm{C}$ for $180 \mathrm{~min}$ showed the $\mathrm{Si}$ core diameter of the SiNWs to be self-limited to about $8 \mathrm{~nm}$. The result shows that the self-limiting oxidation process yields excellent control of the diameter of the Si core of the SiNWs. It is important to control the diameter of the $\mathrm{Si}$ core of the SiNWs at the application stage, since the physical properties of one-dimensional SiNWs closely depend on the Si core diameter. Hence, the self-limiting oxidation process might be one of the most promising methods for controlling the $\mathrm{Si}$ core diameter of the SiNWs.

On the other hand, both Raman shift and peak width of the Si optical phonon peak for the $1000{ }^{\circ} \mathrm{C}$ oxidation case showed an increase, even after oxidation for $180 \mathrm{~min}$. This result means that oxidation still progresses at $1000{ }^{\circ} \mathrm{C}$, i.e., oxygen can overcome the stress and diffuse along the surface oxide layer at this temperature. The compressive stress induced by thermal oxidation at $1000{ }^{\circ} \mathrm{C}$ for $180 \mathrm{~min}$ is roughly estimated to be about $350 \mathrm{MPa}$. According to previous studies on the self-limiting oxidation effect in lithographically formed $\mathrm{Si}$ nanocolumns, ${ }^{22,23}$ the self-limiting oxidation phenomenon occurs only at oxidation temperatures below $950{ }^{\circ} \mathrm{C}$. The temperature dependence of self-limiting oxidation for the SiNWs formed by the bottom-up process of laser ablation is in accordance with the case for lithographically formed Si nanocolumns.

\section{SUMMARY}

Phonon confinement and the self-limiting oxidation effect of SiNWs synthesized by laser ablation were investigated. The size of the SiNWs was controlled by the synthesis parameters during laser ablation and the subsequent thermal oxidation. The Si optical phonon peak of the SiNWs showed a downshift and an asymmetric broadening, due to the phonon confinement effect, with decreasing Si core diameter of the SiNWs. Thermal oxidation of the SiNWs resulted in a gradual but clearly identifiable shift and broadening of the $\mathrm{Si}$ optical phonon peak with decreasing $\mathrm{Si}$ core diameter of the SiNWs. The promotion of oxidation, however, induces compressive stress in SiNWs, resulting in an upshift of the optical phonon peak. The formation of such a highly stressed oxide decreases the oxidation rate due to the self-limiting oxidation effect.

\section{ACKNOWLEDGMENTS}

This work was partly supported by a Grant-in-Aid for Scientific Research (2005) to Young Scientists (B), (17760003) from the Ministry of Education, Culture, Sports, Science and Technology (MEXT), Japan. TEM observations were also partly supported by the Nanotechnology Support Project of the Ministry of Education, Culture, Sports, Science and Technology (MEXT), Japan.

${ }^{1}$ Y. Cui and C. M. Lieber, Science 291, 851 (2001).

${ }^{2}$ J. F. Wang, M. S. Gudiksen, X. F. Duan, Y. Cui, and C. M. Lieber, Science 293, 1455 (2001).

${ }^{3}$ X. F. Duan, Y. Huang, Y. Cui, J. F. Wang, and C. M. Lieber, Nature (London) 409, 66 (2001).

${ }^{4}$ Y. Cui, Q. Wei, H. Park, and C. M. Lieber, Science 293, 1289 (2001).

${ }^{5}$ T. Arai and M. Tomitori, Appl. Phys. Lett. 86, 073110 (2005).

${ }^{6}$ N. Fukata, T. Oshima, T. Tsurui, S. Ito, and K. Murakami, Sci. Technol. Adv. Mater. 6, 628 (2005).

${ }^{7}$ A. M. Morales and C. M. Lieber, Science 279, 208 (1998).

${ }^{8}$ Y. F. Zhang, Y. H. Tang, N. Wang, D. P. Yu, C. S. Lee, I. Bello, and S. T. Lee, Appl. Phys. Lett. 72, 1835 (1998).

${ }^{9}$ S. T. Lee, N. Wang, and C. S. Lee, Mater. Sci. Eng., A 286, 16 (2000).

${ }^{10}$ N. Fukata, T. Oshima, K. Murakami, T. Kizuka, T. Tsurui, and S. Ito, Appl. Phys. Lett. 86, 213112 (2005).

${ }^{11}$ J. Westwater, D. P. Gosain, and S. Usui, Jpn. J. Appl. Phys., Part 1 36, 6204 (1997) 
${ }^{12}$ Y. Cui, L. J. Lauhon, M. S. Gudiksen, J. Wang, and C. M. Lieber, Appl. Phys. Lett. 78, 2214 (2001).

${ }^{13}$ R. S. Wagner and W. C. Ellis, Appl. Phys. Lett. 4, 89 (1964).

${ }^{14}$ E. I. Givargizov, J. Cryst. Growth 31, 20 (1975).

${ }^{15}$ X. Zhao, C. M. Wei, L. Yang, and M. Y. Chou, Phys. Rev. Lett. 92 236805 (2004)

${ }^{16}$ D. P. Yu et al., Phys. Rev. 59, R2498 (1999).

${ }^{17}$ D. D. D. Ma, C. S. Lee, F. C. K. Au, S. Y. Tong, and S. T. Lee, Science 299, 1874 (2003).

${ }^{18}$ S. Piscanec, M. Cantro, A. C. Ferrari, J. A. Zapien, Y. Lifshitz, S. T. Lee, S. Hofmann, and J. Robertson, Phys. Rev. B 68, 241312 (2003).

${ }^{19}$ S. Bhattachayya and S. Samui, Appl. Phys. Lett. 84, 1564 (2004).
${ }^{20}$ K. W. Adu, H. R. Gutierrez, U. J. Kim, G. U. Sumanasekera, and P. C. Eklund, Nano Lett. 5, 400 (2005).

${ }^{21}$ N. Fukata, T. Oshima, N. Okada, T. Kizuka, T. Tsurui, S. Ito, and K. Murakami, Physica B 376-377, 864 (2006).

${ }^{22}$ H. I. Liu, D. K. Biegelsen, F. A. Ponce, N. M. Johnson, and R. F. W. Pease, Appl. Phys. Lett. 64, 1383 (1994).

${ }^{23}$ H. Fukuda, J. L. Hoyt, M. A. McCord, and R. F. W. Pease, Appl. Phys. Lett. 70, 333 (1997).

${ }^{24}$ H. Richter, Z. P. Wang, and L. Ley, Solid State Commun. 39, 625 (1981).

${ }^{25}$ I. H. Campbell and P. M. Fauchet, Solid State Commun. 58, 739 (1986).

${ }^{26}$ T. P. Mernagh and L. G. Liu, J. Phys. Chem. Solids 52, 507 (1991). 